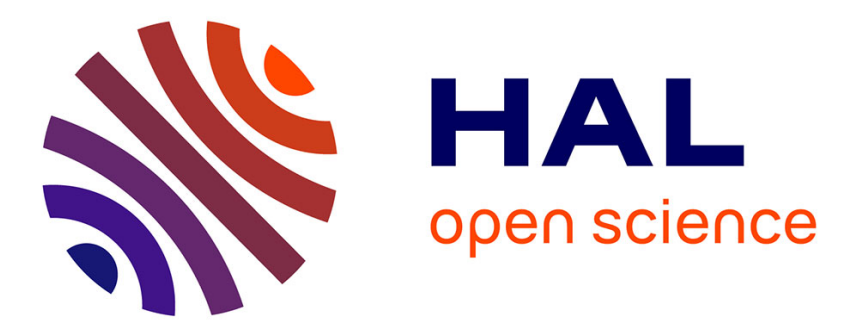

\title{
Force chain collapse as grain column buckling in granular materials
}

François Nicot, Hao Xiong, Antoine Wautier, Jean Lerbet, Félix Darve

\section{To cite this version:}

François Nicot, Hao Xiong, Antoine Wautier, Jean Lerbet, Félix Darve. Force chain collapse as grain column buckling in granular materials. Granular Matter, 2017, 19 (2), pp.12-. 10.1007/s10035-0170702-0 . hal-01486649

\author{
HAL Id: hal-01486649 \\ https://hal.science/hal-01486649
}

Submitted on 16 Oct 2017

HAL is a multi-disciplinary open access archive for the deposit and dissemination of scientific research documents, whether they are published or not. The documents may come from teaching and research institutions in France or abroad, or from public or private research centers.
L'archive ouverte pluridisciplinaire HAL, est destinée au dépôt et à la diffusion de documents scientifiques de niveau recherche, publiés ou non, émanant des établissements d'enseignement et de recherche français ou étrangers, des laboratoires publics ou privés. 


\title{
Force chain collapse as grain column buckling in granular materials
}

\author{
François Nicot $^{1} \cdot$ Hao Xiong ${ }^{1} \cdot$ Antoine Wautier $^{1,4,5} \cdot$ Jean Lerbet $^{2} \cdot$ Félix Darve $^{3}$
}

\begin{abstract}
Granular materials react under external loading by self-organizing the topology of the grain assembly. Thus, quasi-linear grain patterns, known as force chains, develop within the assembly to carry the main part of the external loading. The stability of these grain patterns controls the strength of the material, under given loading conditions. This manuscript investigates the mechanical behavior of such grain columns and exhibits some instability modes that can occur even when the local behavior at the contact between grains is purely elastic. First, an analytical approach is developed, and the ability of a grain column to collapse is demonstrated. Then this result is confirmed from numerical simulations performed using a discrete element method.
\end{abstract}

Keywords Stress · Instability · Collapse · Failure · Secondorder work · Granular materials · Microstructure $\cdot$ Force chains

François Nicot

francois.nicot@irstea.fr

1 Université Grenoble-Alpes, IRSTEA, ETNA - Geomechanics Group, Grenoble, France

2 Laboratoire IBISC, Université d'Evry Val d'Essonne, UFR Sciences et Technologie, Evry, France

3 Université Grenoble-Alpes, Lab. Sols Solides Structures Risques, UJF-INPG-CNRS, Grenoble, France

4 AgroParisTech-ENGREF, 19 avenue du Maine, 75732 Paris, France

5 IRSTEA, UR RECOVER, 3275 Rte Cézanne, CS 40061, 13182 Aix-en-Provence Cedex 5, France

\section{Introduction}

Material instabilities and geometric instabilities have classically been distinguished. The first basically stem from an unstable material behavior and occur experimentally in initially homogeneous samples for some loading conditions [12]. On the other hand, geometric instabilities are induced by specific geometries of the body or by arrangements of the discrete elements constituting the mechanical system, while the constitutive behavior of these elements can be perfectly stable [23].

Material instabilities are present in granular materials as, for example, the softening behavior of dense sands or the formation of shear bands as plastic strain localization consequences. All these material instabilities leading to failure by divergence instability (keeping in mind that flutter instability is also possible; [14]) are described by a single criterion: the so-called second-order work criterion [31]. Many studies have investigated this question from experimental [7], theoretical $[8,19,20]$ and numerical viewpoints through the finite element method (FEM) [11] and the discrete element method (DEM) [25]. However, it was experimentally observed 50 years ago [6] and verified from a discrete numerical approach [24] that inside a granular assembly force chains are always present, transferring the forces applied to the sample boundaries through the granular assembly. More recently, thanks to DEM analyses, another kind of granular mesostructure has been observed, which links contacting grains through closed cycles, the so-called grain loops or grain cycles $[10,29,30,34]$. These loops, which can involve different numbers of grains, seem to be less prone to transfer forces than chains. They are more involved in the deformation features of the granular body. Thus, inside a macroscopically homogeneous granular sample, some internal structures 
develop by self-organization of the granular assembly over a mechanical loading: force chains and grain loops.

It can be expected that these internal meso-structures could develop their own instability mechanisms - here through geometric instability phenomena [28]. The purpose of this paper is to consider if such instabilities can develop for force chains, while loops have been investigated previously in other papers [22]. Throughout the manuscript, a rather simple intergranular contact law is considered: the interactions between grains are assumed to be purely elastic. The stability of discrete elastic systems has been a classical question for half a century $[2,33]$ and it has been shown that these systems can develop geometric instabilities even for purely elastic interactions between their components. Some "paradoxical" effects have even been observed in nonconservative elasticity. Indeed, in this last case, the stiffness matrix is nonsymmetric. Let us recall that, in elastoplasticity, the plastic nonassociative behavior leads to a nonsymmetric constitutive matrix and thus to bifurcation states strictly within the plastic limit surface for certain loading paths. These unstable states are accurately described by the second-order work criterion [26]. In exactly the same way, this nonsymmetry in discrete nonconservative elastic systems induces "paradoxical" bifurcations for certain kinematic constraints, also accurately predicted by the second-order work criterion [15]. Thus, within the same framework of elastic discrete systems, the questions of granular force chain stability and related collapse mechanisms are considered in this paper.

Before any stability analysis, a first question can be raised: how can the interaction elastic law between two contacting spheres be properly defined? The model proposed by Cundall [5] is based on a normal elastic law relating the normal component of the incremental contact force to the normal relative incremental displacement and on a tangential elastic law defined in the same way. This kind of law has proved to be efficient in innumerable DEM modeling, which has been compared successfully to experimental results obtained in $2 \mathrm{D}$ conditions with circular piled cylinders and in 3D conditions with spherical grains. However, within the perspective of analytical analyses of force chain stability, no expression of the interaction elastic law in an integrated or global form generally exists. Thus, problems arise here since Cundall's incremental elastic law can be integrated only with additional assumptions, which are in fact debatable. As an example of these difficulties, let us briefly mention the fact that the incremental tangential displacement cannot be generally integrated in presence of normal granular interpenetrations, which are, however, necessarily induced by the normal elastic law [22]. More precisely, following these additional assumptions, the interaction elastic law can take different forms and, roughly speaking, it can be hyperelastic or hypoelastic. For bifurcation analyses, this point is crucial, since in hyperelasticity the stiffness matrix will be symmetric because of the existence of a global elastic potential and will be non-symmetric in hypoelasticity without any potential $[1,9,32]$. This general discussion is not considered in this paper and it will be treated in a further paper. Here a hypoelastic framework is detailed in Subsect. 2.1, showing the nonconservativeness of the local intergranular law essentially because of the normal grain deformability.

The paper is structured logically after this first subsection on the constitutive formalism. According to the analytical model, some limit states are obtained in Sect. 2.2. Then these limit states are studied, first from the analytical formalism perspective in Sect. 3.1 and from a DEM modelling in Sect. 3.2. A comparison is presented and analyzed in the closure discussion in the fourth section.

\section{Modeling a grain column mechanically}

\subsection{Geometrical setting and governing equations}

We consider a linear-like assembly of $n$ spherical particles in contact, as depicted in Fig. 1. The spheres have the same radius $r$. The geometry of the assembly is described by means of the distribution of deflecting angles $\alpha_{i}$ and branch distances $l_{i}$ between the two adjoining particles ' $i$ ' and ' $i-1$ '. The elementary assembly is assumed to be loaded by an axial force $F_{1}$ applied to grains ' 1 ' and ' $n$ ' located at the ends of the chain (grain ' $n$ ' is reputed to be fixed with respect to a suitable Galilean frame), and a set of $n$ lateral forces $G_{i}$ oriented perpendicularly to the axial force $F_{1}$. The lateral balance condition imposes that $\sum_{i=1}^{n} G_{i}=0$. This system is believed to model a force chain composed of $n$ particles. The lateral forces model the confining effect directed by the neighboring grains (in a granular assembly). This model differs from what was developed by Tordesillas and Muthuswamy [28], where the authors modeled the confining effect applied to the grain column by a series of lateral springs.

The purpose of this section is to investigate the mechanical behavior of this assembly, by focusing on the possible occurrence of instability modes.

The length of the force chain is denoted $L_{1}$, and corresponds to the axial distance between particles ' 1 ' and ' $n$ '. It can be written:

$L_{1}=\sum_{i=1}^{n-1} l_{i} \cos \alpha_{i}$

Prior to any loading, any branch length verifies $l_{i}=2 r$, and the geometrical configuration of the force chain is entirely described by the initial values $\alpha_{i, o}$ of the deflecting angles. Applying an external loading $\left(F_{1}, G_{1}, \ldots G_{n}\right)$ changes the geometry of the assembly. Assuming the spheres are rigid 


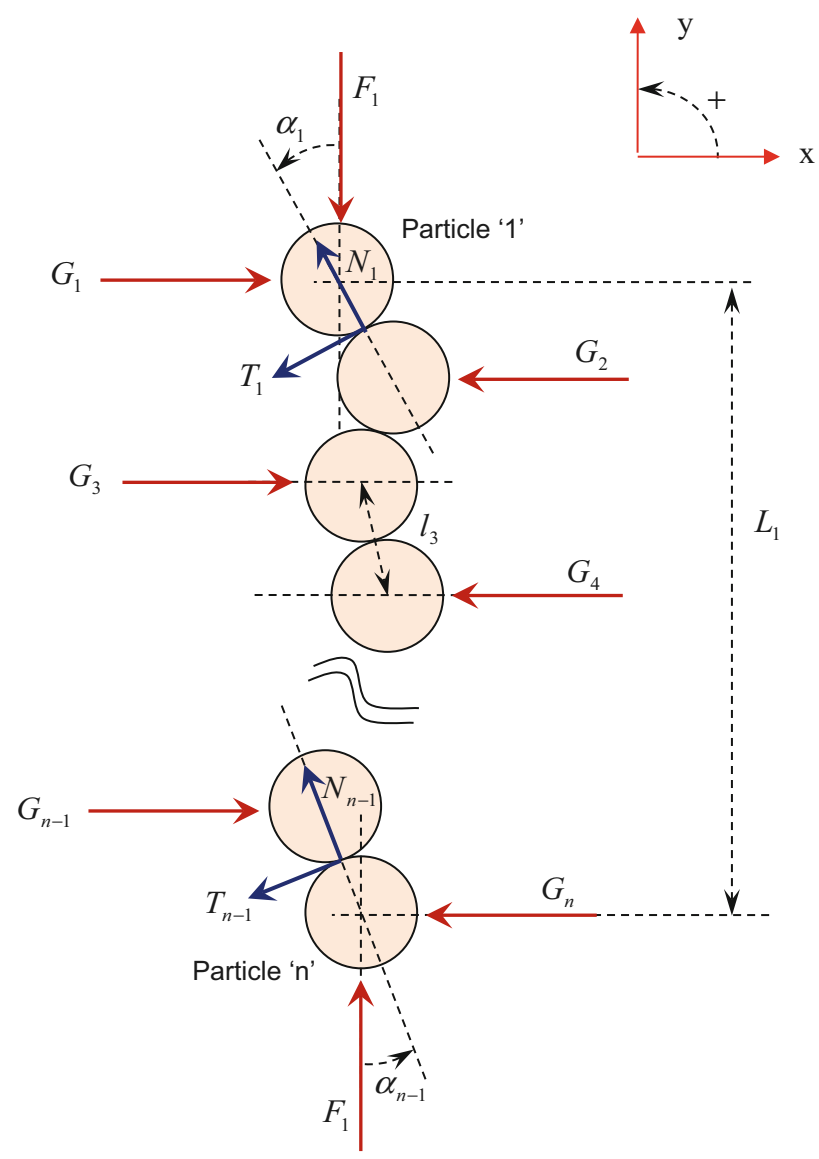

Fig. 1 The linear-like pattern. Geometrical model and notations

bodies, the change in geometry is related to the relative motion between the spheres. The normal component of the relative displacement between particles ' $i$ ' and ' $i-1$ ' is denoted $u_{i}^{n}$, whereas the tangential counterpart is denoted $u_{i}^{t}$. This relative displacement directs a contact force between the adjoining grains; $N_{i}$ is the normal component and $T_{i}$ is the tangential component. Furthermore, the rotation $\theta_{i}$ of any particle induced by the torque $M_{i}=r\left(T_{i+1}-T_{i}\right)$ will be disregarded. This situation corresponds to an infinite rolling resistance, which can account for nonspherical particles with surface irregularities (as for granular soils, for example).

At any stage of the loading, the following balance equations hold:

For particle ' 1 '

$$
\begin{aligned}
& F_{1}=N_{1} \cos \alpha_{1}-T_{1} \sin \alpha_{1} \\
& G_{1}=N_{1} \sin \alpha_{1}+T_{1} \cos \alpha_{1}
\end{aligned}
$$

For particle ' $i$ ' $(2 \leq i \leq n-1)$

$$
\begin{aligned}
N_{i-1} & \cos \alpha_{i-1}-T_{i-1} \sin \alpha_{i-1} \\
= & N_{i} \cos \alpha_{i}-T_{i} \sin \alpha_{i} \\
G_{i}= & N_{i} \sin \alpha_{i}+T_{i} \cos \alpha_{i}
\end{aligned}
$$

$$
-N_{i-1} \sin \alpha_{i-1}-T_{i-1} \cos \alpha_{i-1}
$$

For particle ' $n$ '

$$
\begin{aligned}
& F_{1}=N_{n-1} \quad \cos \alpha_{n-1}-T_{n-1} \sin \alpha_{n-1} \\
& G_{n}=-N_{n-1} \sin \alpha_{n-1}-T_{n-1} \cos \alpha_{n-1}
\end{aligned}
$$

It is worth noting that Eqs. (2), (3) and (4) can be merged into the following relations $(1 \leq i \leq n-1)$ :

$$
\sum_{j=1}^{i} G_{j}=N_{i} \sin \alpha_{i}+T_{i} \cos \alpha_{i}
$$

$N_{i} \cos \alpha_{i}-T_{i} \sin \alpha_{i}=F_{1}$

The local behavior is assumed to be purely elastic and can be expressed under the following rate formalism, which introduces a normal elastic stiffness $k_{n}$ and a tangential elastic stiffness $k_{t}$, both constant:

$$
\begin{aligned}
& \dot{N}_{i}=-k_{n} \dot{u}_{i}^{n} \\
& \dot{T}_{i}=-k_{t} \dot{u}_{i}^{t}
\end{aligned}
$$

The standard soil mechanics sign convention is adopted throughout the text: the forces are counted positive in compression.

The balance equations can be written under a rate form. After differentiating Eq. (6), as $\dot{u}_{i}^{n}=\dot{l}_{i}$ and $\dot{u}_{i}^{t}=l_{i} \dot{\alpha}_{i}$ (see [22], for a detailed discussion on the kinematic description of the contact between two spheres), the following can be obtained:

$$
\begin{aligned}
\dot{F}_{1}= & -k_{n} \dot{l}_{i} \cos \alpha_{i}+k_{t} l_{i} \dot{\alpha}_{i} \sin \alpha_{i} \\
& -\left(N_{i} \sin \alpha_{i}+T_{i} \cos \alpha_{i}\right) \dot{\alpha}_{i}
\end{aligned}
$$

And by virtue of Eq. (5):

$\dot{F}_{1}=-k_{n} \dot{l}_{i} \cos \alpha_{i}+k_{t} l_{i} \dot{\alpha}_{i} \sin \alpha_{i}-\sum_{j=1}^{i} G_{j} \dot{\alpha}_{i}$

As for $i=1$, Eq. (9) reads:

$\dot{F}_{1}=-k_{n} \dot{l}_{1} \cos \alpha_{1}+k_{t} l_{1} \dot{\alpha}_{1} \sin \alpha_{1}-G_{1} \dot{\alpha}_{1}$

We finally obtain, for $1 \leq i \leq n-1$ :

$$
\begin{aligned}
& -k_{n} \dot{l}_{1} \cos \alpha_{1}+\left(k_{t} l_{1} \sin \alpha_{1}-G_{1}\right) \dot{\alpha}_{1}=-k_{n} \dot{l}_{i} \cos \alpha_{i} \\
& +\left(k_{t} l_{i} \sin \alpha_{i}-\sum_{j=1}^{i} G_{j}\right) \dot{\alpha}_{i}
\end{aligned}
$$


Moreover, differentiating Eq. (5) gives, with the help of Eq.

(6):

$$
\sum_{j=1}^{i} \dot{G}_{j}=-k_{n} \dot{l}_{i} \sin \alpha_{i}-k_{t} l_{i} \dot{\alpha}_{i} \cos \alpha_{i}+F_{1} \dot{\alpha}_{i}
$$

Thereafter, we particularize the loading to the following case: starting from an initial geometrical configuration $\left(\alpha_{i, o}\right)$, the lateral forces $G_{i}$ are kept constant $\left(\dot{G}_{i}=0\right)$, while the axial loading is monotonously increased: $\dot{L}_{1}=$ const(negative). $L_{1}$ is therefore the control parameter.

Then, Eq. (12) gives, for $1 \leq i \leq n-1$ :

$\dot{l}_{i}=\frac{F_{1}-k_{t} l_{i} \cos \alpha_{i}}{k_{n} \sin \alpha_{i}} \dot{\alpha}_{i}$

Combining Eq. (13) with Eq. (11) yields, for $2 \leq i \leq n-1$ :

$$
\begin{aligned}
& \left.\left(F_{1}-k_{t} l_{i} \cos \alpha_{i}\right) \cot \alpha_{i}+\sum_{j=1}^{i} G_{j}-k_{t} l_{i} \sin \alpha_{i}\right) \dot{\alpha}_{i} \\
& =\left(\left(F_{1}-k_{t} l_{1} \cos \alpha_{1}\right) \cot \alpha_{1}+G_{1}-k_{t} l_{1} \sin \alpha_{1}\right) \dot{\alpha}_{1}
\end{aligned}
$$

For conciseness, Eqs. (13) and (14) can be expressed as:

$A_{i} \dot{\alpha}_{i}=A_{1} \dot{\alpha}_{1}$, for $2 \leq i \leq n-1$

$\dot{l}_{i}=B_{i} \dot{\alpha}_{i}$, for $1 \leq i \leq n-1$

with $B_{i}=\frac{F_{1}-k_{t} l_{i} \cos \alpha_{i}}{k_{n} \sin \alpha_{i}}$ and $A_{i}=\left(F_{1} \cos \alpha_{i}-k_{t} l_{i}\right) /$ $\sin \alpha_{i}+\sum_{j=1}^{i} G_{j}$.

Furthermore, differentiating Eq. (1) yields:

$\dot{L}_{1}=\sum_{i=1}^{n-1}\left(B_{i} \cos \alpha_{i}-l_{i} \sin \alpha_{i}\right) \dot{\alpha}_{i}$

Given any value $\dot{L}_{1}$, the $n-1$ geometrical parameters $\left(\alpha_{1}, \ldots \alpha_{n-1}\right)$ can be determined from Eqs. (15) and (17). Thus, the following linear system can be derived:

$\left[\begin{array}{cccc}C_{1} & C_{2} & \cdots & C_{n-1} \\ A_{1} & -A_{2} & \cdots & 0 \\ \vdots & \vdots & \ddots & \vdots \\ A_{1} & 0 & \cdots & -A_{n-1}\end{array}\right]\left[\begin{array}{c}\dot{\alpha}_{1} \\ \dot{\alpha}_{2} \\ \vdots \\ \dot{\alpha}_{n-1}\end{array}\right]=\left[\begin{array}{c}\dot{L}_{1} \\ 0 \\ \vdots \\ 0\end{array}\right]$

with $C_{i}=B_{i} \cos \alpha_{i}-l_{i} \sin \alpha_{i}$. The existence of a unique solution is guaranteed if $\operatorname{det} \mathbf{H} \neq 0$, with:

$\mathbf{H}=\left[\begin{array}{cccc}C_{1} & C_{2} & \cdots & C_{n-1} \\ A_{1} & -A_{2} & \cdots & 0 \\ \vdots & \vdots & \ddots & \vdots \\ A_{1} & 0 & \cdots & -A_{n-1}\end{array}\right]$
$\mathbf{H}$ stands as a matrix that relates the evolution of the kinematical parameters of the system (angles $\alpha_{i}$ ) to the evolution of the control parameter $L_{1}$. It is worth mentioning that matrix $\mathbf{H}$ is not intrinsic but depends on the loading path considered (here, lateral forces are imposed constant).

$$
\text { As } \operatorname{det} \mathbf{H}=(-1)^{n-1} \sum_{i=1}^{n-1}\left(C_{i} \prod_{\substack{j=1 \\ j \neq i}}^{n-1} A_{j}\right) \text {, it appears that }
$$
$\operatorname{det} \mathbf{H}=0$ as soon as any two terms $A_{i}$ are zero.

\subsection{Existence of a limit state}

The resulting axial force $F_{1}$ is obtained from Eqs. (9) and (13). After simplification, the following relation can be inferred:

$\dot{F}_{1}=-A_{1} \dot{\alpha}_{1}$

Taking advantage of Eqs. (15), (20) can also be expressed as:

$(\forall i=1, \ldots n-1) \quad \dot{F}_{1}=-A_{i} \dot{\alpha}_{i}$

As a result, Eq. (21) shows that a limit state $\left(\dot{F}_{1}=0\right)$ can be reached along a given loading path if one any term $A_{i}$ vanishes. Thus, the condition for having a limit state reads:

$\exists i \in[1, \ldots n-1] / F_{1} \cos \alpha_{i}+\sum_{j=1}^{i} G_{j} \sin \alpha_{i}-k_{t} l_{i}=0$

If only one term vanishes, say $A_{j}$, the system has a unique solution:

$\dot{\alpha}_{j}=\frac{\dot{L}_{1}}{B_{j} \cos \alpha_{j}-l_{j} \sin \alpha_{j}}$ and $(\forall i \neq j) \dot{\alpha}_{i}=0$

This can be regarded as localized buckling, with a strong deviation effect between particles ' $j$ ' and ' $j+1$ '. Since $\operatorname{det} \mathbf{H}$ does not vanish, Eq. (18) has a unique solution. The system therefore continues to deform continuously, under the control of the kinematic variable $L_{1}$. With this control, this limit state is not associated with any effective failure. However, if the loading control is changed into a force control (an additional axial loading is prescribed), then an abrupt collapse is expected. The system does not have any geometrical configuration that can balance the external loading.

If more than one term, say $m$ terms $A_{j_{1}}, \ldots A_{j_{m}}$, vanish, the system given by Eq. (18) does not have a unique solution (except if symmetry conditions hold): $\left(\forall i \neq j_{1}, \ldots j_{m}\right) \dot{\alpha}_{i}=$ 0 , and $\dot{\alpha}_{j_{1}}, \ldots \dot{\alpha}_{j_{m}}$ are undetermined under the prescription $\sum_{k=1}^{m}\left(B_{j_{k}} \cos \alpha_{j_{k}}-l_{j_{k}} \sin \alpha_{j_{k}}\right) \dot{\alpha}_{j_{k}}=\dot{L}_{1}$. 
Table 1 Numerical simulation: constitutive and general parameters

\begin{tabular}{llll}
\hline$n$ & $r(\mathrm{~m})$ & $k_{n}(\mathrm{kN} / \mathrm{m})$ & $k_{t}(\mathrm{kN} / \mathrm{m})$ \\
\hline 5 & 0.1 & 1000 & 400 \\
\hline
\end{tabular}

Table 2 Numerical simulation: initial geometrical configuration $\left(\alpha_{i, o}\right)$

\begin{tabular}{llll}
\hline$\alpha_{1, o}\left({ }^{\circ}\right)$ & $\alpha_{2, o}\left({ }^{\circ}\right)$ & $\alpha_{3, o}\left({ }^{\circ}\right)$ & $\alpha_{4, o}\left({ }^{\circ}\right)$ \\
\hline 9 & -23 & 23 & -9 \\
\hline
\end{tabular}

Table 3 Numerical simulation: lateral forces $G_{i}$

\begin{tabular}{lllll}
\hline$G_{1} /\left(2 r k_{n}\right)$ & $G_{2} /\left(2 r k_{n}\right)$ & $G_{3} /\left(2 r k_{n}\right)$ & $G_{4} /\left(2 r k_{n}\right)$ & $G_{5} /\left(2 r k_{n}\right)$ \\
\hline 1 & -1.5 & 1 & -1.5 & 1 \\
\hline
\end{tabular}

\section{Numerical inspection}

\subsection{Inspection using the analytical model}

To illustrate these results, the governing Eqs. (18) and (21) were solved numerically. The simulation is run using the parameters reported in Tables 1, 2 and 3. The lateral forces $G_{i}$ are kept constant $\left(\dot{G}_{i}=0\right)$, while the axial loading is monotonously increased: $\dot{L}_{1}=$ const (negative).

The evolution of the axial force $\left(F_{1}\right)$ as a function of the axial strain $\left(1-L_{1} / L_{1 o}\right)$ is reported in Fig. 2. After an increasing phase, the axial force reaches a peak (9.56 $\mathrm{kN}$ ), then follows a descending branch. At the peak (corresponding to a limit state), the system is clearly unstable. Any additional axial force applied to the system will lead to the abrupt collapse of the chain. As seen in Fig. 3, term $A_{2}$ vanishes exactly at the point where the axial force reaches the peak (for an axial strain of 18\%), whereas $A_{1}$ does not vanish (but reaches an extremum). For symmetry reasons, $A_{1}=-A_{4}$ and $A_{2}=-A_{3}$.

The change in the geometrical configuration of the chain is given in Fig. 4, with the evolution of the $\alpha_{i}$ angles. For symmetry reasons, $\alpha_{1}=-\alpha_{4}$ and $\alpha_{2}=-\alpha_{3}$.

Figure 5 shows the geometrical configuration of the chain, prior to loading (left) and at the end of loading (right). It is worth noting that the chain experiences a sharp localized collapse (called buckling) with significant grain rearrangement (grain translation) in the central part of the column between grains 2 and 3, and grains 3 and 4 .

Depending on the initial configuration and the loading conditions, this localization can occur at different places on the chain. To investigate this question, we must return to Eq. (22), which gives the condition for a limit state to occur.
Equation (22) can be expressed as:

$F_{1}=\frac{k_{t} l_{i}}{\cos \alpha_{i}}-\sum_{j=1}^{i} G_{j} \tan \alpha_{i}$

where the localized buckling of the column will occur in the first branch ' $i$ ' (joining grains $i$ and $i+1$ ) that fulfills Eq. (24).

As the axial force $F_{1}$ increases over loading, the localized buckling occurs in the branch ' $i$ ' corresponding to the smallest term $\chi_{i}=\frac{k_{t} l_{i}}{\cos \alpha_{i}}-\sum_{j=1}^{i} G_{j} \tan \alpha_{i}$. The lower the tangent stiffness and the branch length (scaling to the radius of the particles), and the larger $\sum_{j=1}^{i} G_{j}$ (or smaller, if negative), the lower the magnitude of these terms is. Thus, the lateral forces direct a counterintuitive effect by promoting the occurrence of a limit state, whereas they apply lateral confining (modeling, for example, the interactions with the other grains in a granular assembly). In the previous example, the different terms $\chi_{j}$ can be assessed at the initial state:

$$
\begin{aligned}
& \chi_{1}=\chi_{4}=\frac{2 r k_{n}}{\cos \alpha_{1,0}}\left(0.4-\sin \alpha_{1,0}\right) \approx 0.49 r k_{n} \\
& \chi_{2}=\chi_{3}=\frac{2 r k_{n}}{\cos \alpha_{2,0}}\left(0.4+0.5 \sin \alpha_{2,0}\right) \approx 0.44 r k_{n}
\end{aligned}
$$

Ignoring the evolution of angles $\alpha_{1}$ and $\alpha_{2}$, this analysis shows that $\chi_{2}<\chi_{1}$, therefore justifying that the localized buckling occurs in the branches $i=2$ and $i=3$, between grain 3 and grains 2 and 4 .

By changing the initial geometrical configuration (Table 4), one can expect that the system will collapse with a sharp localization at the extremities of the column as:

$$
\begin{aligned}
& \chi_{1}=\chi_{4}=\frac{2 r k_{n}}{\cos \alpha_{1,0}}\left(0.4-\sin \alpha_{1,0}\right) \approx 0.29 r k_{n} \\
& \chi_{2}=\chi_{3}=\frac{2 r k_{n}}{\cos \alpha_{2,0}}\left(0.4+0.5 \sin \alpha_{2,0}\right) \approx 0.56 r k_{n}
\end{aligned}
$$

As observed in Fig. 6, a limit state is reached at $8 \%$ axial strain. At this state, unlike in the previous case, the term $A_{1}$ vanishes, whereas $A_{2}$ reaches a minimum value (Fig. 7). Moreover, Figs. 8 and 9 show that the deformation within the chain localizes at the extremities of the chain, within the branches $i=1$ and $i=4$. Both angles $\alpha_{1}$ and $\alpha_{4}$ increase significantly after the limit state is reached, whereas angles $\alpha_{2}$ and $\alpha_{3}$ remain more or less constant. 
Fig. 2 Axial force versus axial strain

Fig. 3 Terms $A_{1}$ and $A_{2}$ versus axial strain

Fig. 4 Angles $\alpha_{1}, \alpha_{2}, \alpha_{3}$ and $\alpha_{4}$ versus axial strain
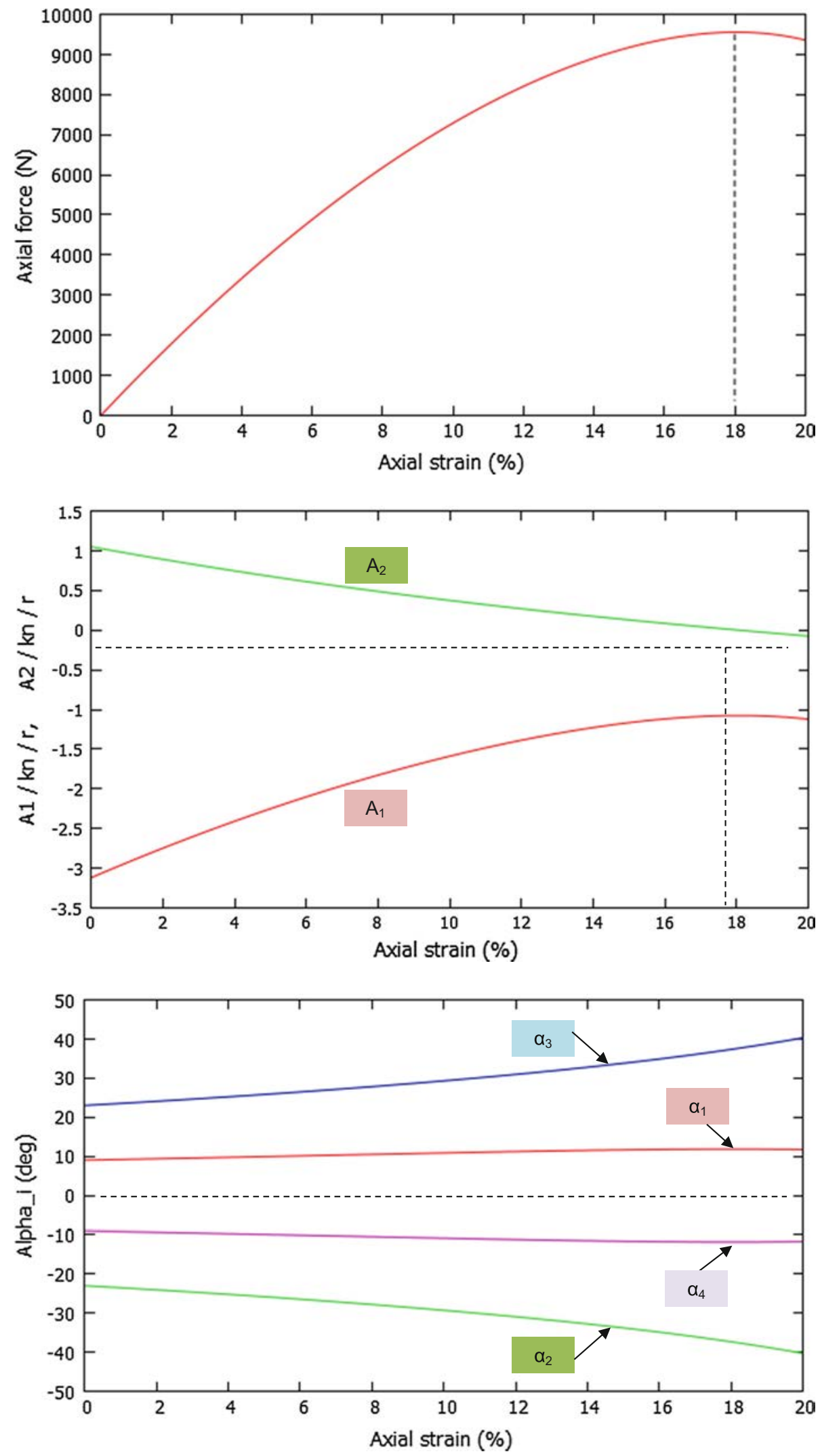


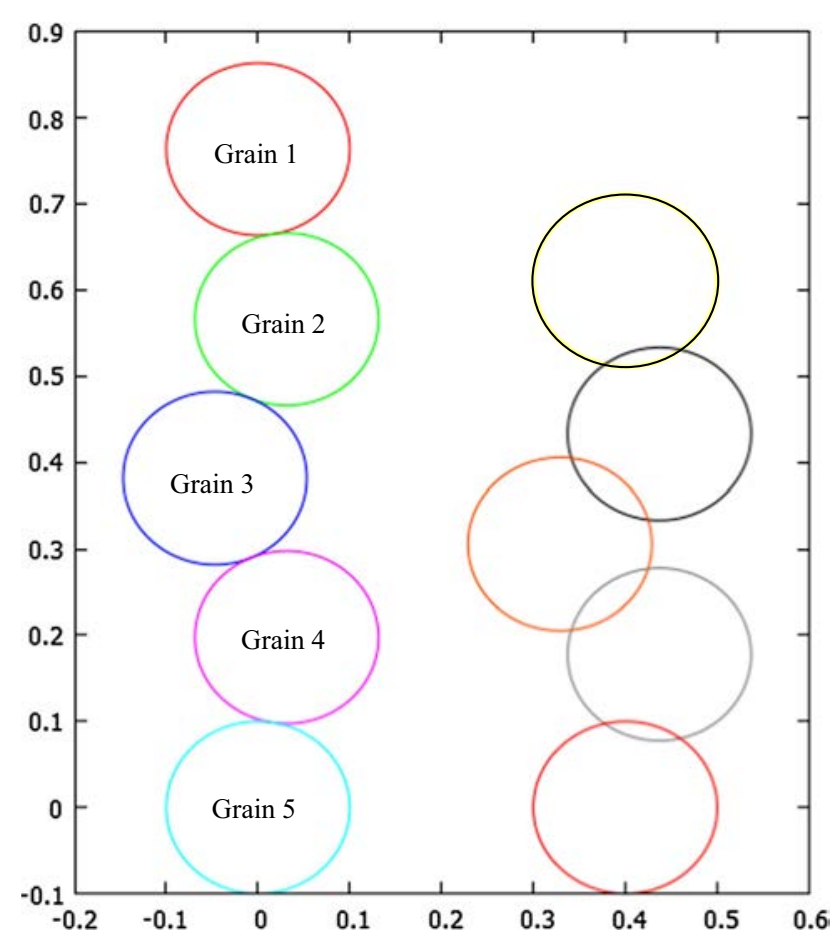

Fig. 5 Geometrical configuration of the chain before loading (left $)$ and after loading (right)

Table 4 Numerical simulation: initial geometrical configuration $\left(\alpha_{i, o}\right)$

\begin{tabular}{llll}
\hline$\alpha_{1, o}\left(^{\circ}\right)$ & $\alpha_{2, o}\left(^{\circ}\right)$ & $\alpha_{3, o}\left(^{\circ}\right)$ & $\alpha_{4, o}\left({ }^{\circ}\right)$ \\
\hline 15 & -15 & 15 & -15 \\
\hline
\end{tabular}

\subsection{Inspection from a discrete element model}

Complementary to the analytical approach developed in Sect. 2, the grain column problem is approached using a Discrete Element Method [5] implemented in YADE software $[13,27]$ in order to recover the buckling of the force chain predicted analytically.

As shown in Fig. 10, five spherical particles of identical radii $r$ are generated in the $(x, y)$ plane in the same geometrical configuration as presented in Table 2. To keep the problem two-dimensional even though YADE software is designed for 3D simulations, displacement along the $z$-axis of the whole system is prevented. Moreover, the rolling effect is dismissed by preventing the particles from rolling. We do insist that a simplified model was considered to investigate the mechanical behavior of grain columns and to exhibit instability modes that can occur even when the local behavior at the contact between grains is purely elastic. It should be emphasized that the absence of grain rotation is rather stabilizing, restricting the occurrence of instability modes.

As introduced in Sect. 2.1, the inter-particle contact is set to be purely elastic with both normal and tangent contact forces described by the force-displacement relations recalled in Eqs. (7a) and (7b). The normal stiffness $k_{n}$ and the tangential stiffness $k_{t}$ (Fig. 10, right) are set according to the parameters reported in Table 5. Likewise, the lateral forces $\left(G_{1}\right.$ to $\left.G_{4}\right)$ are set equal to the values given in Table 3.

Contrary to the analytical approach where the problem was described from a differential formulation, the initial normal and tangential displacements cannot be dismissed in this DEM approach in order to ensure the initial equilibrium prior to the application of any vertical displacement. These displacements were computed using Eqs. (5), (6), (7a) and (7b) and imposed at each of the four grain contacts. If the normal displacement at contact $i$ corresponds to a geometrical grain interpenetration as $l_{i}=2 r+u_{i}^{n}$, the tangential displacement at contact $i$ is a hidden variable in the DEM code updated at each time increment $d t$ as $d u_{i}^{t}=l_{i} \dot{\alpha}_{i} d t$.

From this initial equilibrium position, the testing procedure used previously is applied by blocking the $y$ coordinate of the bottom grain while moving the top grain step by step in the downward direction until the axial strain reaches $20 \%$. During this strain-controlled procedure, the top and bottom grains are left free to move along the $x$ direction.

Since the DEM solves Newton's second law, the response of the system is inherently dynamic. However, a quasi-static response of the grain column can be reached providing that:

- the incremental displacements imposed on the top grain are very small compared to the grain radii;

- the system is left free to stabilize around an equilibrium position before applying any new displacement increment.

During this testing procedure, the axial force $F_{1}$ is recorded and its evolution is plotted in terms of the axial strain in Fig. 11. As done for the analytical model, the geometrical evolution of the grain column, characterized by the deflection angles $\alpha_{1}, \alpha_{2}, \alpha_{3}$ and $\alpha_{4}$, is plotted in Fig. 12. Overall, the evolution of both the axial force and the four deflecting angles are found to be very similar to the analytical curves given in Figs. 2 and 4. As observed from the analytical model, the stress-strain curve passes through a peak at $18 \%$ axial strain. The system is therefore thought to be unstable.

To investigate the collapse of the grain column as a consequence of the instability of the system, the control is switched to a force control after $20 \%$ strain (dotted line in Fig. 11). The vertical displacement $y$ of the top grain is reset free and a constant downward axial force $F_{1}^{\text {ext }}$ is applied such that $F_{1}^{e x t}=F_{1}^{20 \%}+d F$ where $F_{1}^{20 \%}$ is the axial force obtained at $20 \%$ strain (end of the displacement-controlled phase) and $d F=0.005 F_{1}^{20 \%}$. At $20 \%$ axial strain, the system is incapable of withstanding the very small additional force $d F$ and a monotonous increase in axial strain is observed in Fig. 11 before the occurrence of localized buckling at $23.5 \%$ axial 


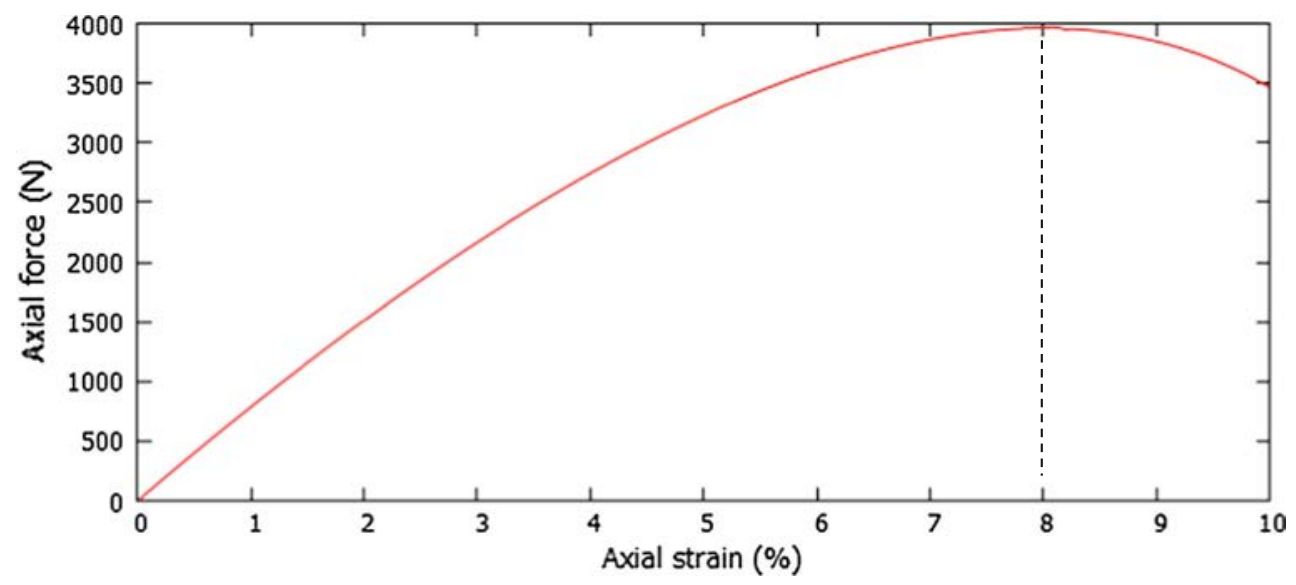

Fig. 6 Axial force versus axial strain

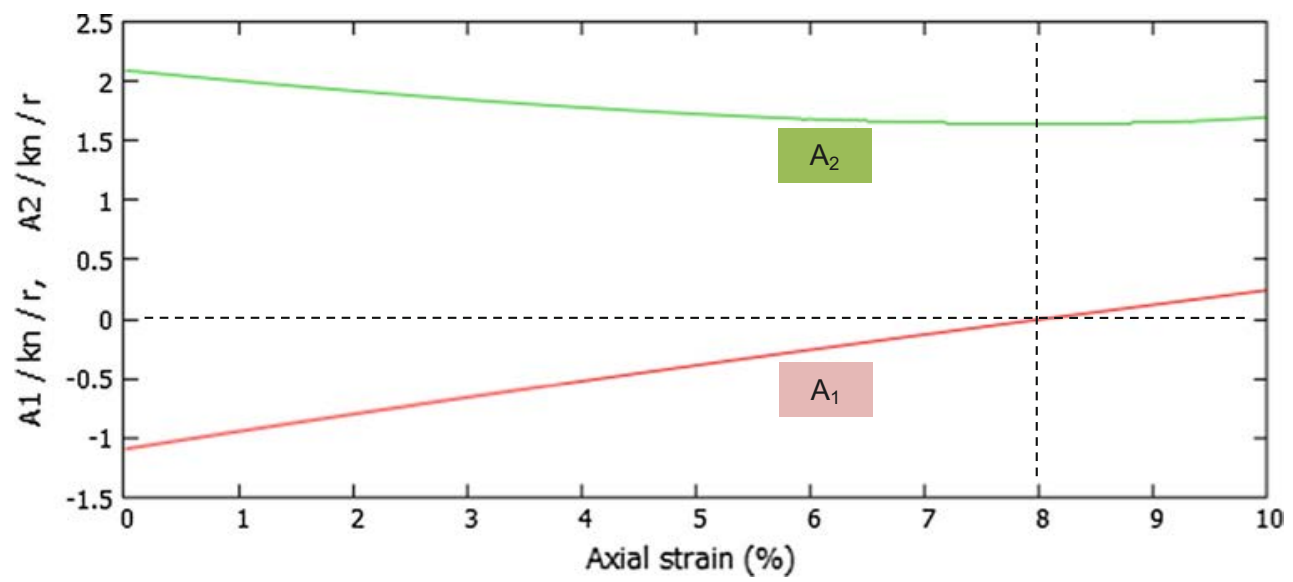

Fig. 7 Terms $A_{1}$ and $A_{2}$ versus axial strain

strain. As mentioned in (Nicot et al. [21]; Nguyen et al. [16]), the occurrence of failure of a given quasi-static system should be linked to a transition toward a dynamic regime characterized by an outburst of kinetic energy. The time evolution of both the axial strain and the kinetic energy during the forcecontrolled phase is shown in Fig. 13.

At first, the axial strain increases more or less linearly while the kinetic energy remains very small. After approximately $30 \mathrm{~s}$, the kinetic energy increases dramatically until an abrupt collapse of the chain occurs, resulting from the loss of contact between several grains. This confirms the unstable state of the system predicted by the analytical approach when the so-called softening regime (descending branch of the axial force, after the peak) is reached. This marks the bifurcation from a quasi-static regime to a dynamical regime $[17,18,21]$. This dynamical regime, which was not within the scope of the static model introduced in Sect. 2, is successfully described here by the discrete element formulation of the problem.

As introduced in Sect. 2, the determinant $\operatorname{det} \mathbf{H}$ stands as the key failure indicator of the grain column given that it should vanish as soon as the column becomes unstable. The evolution of this determinant is shown in Fig. 14 for the two-phase testing procedure introduced in this subsection. As expected, its absolute value decreases until reaching zero at the peak, highlighting that this limit state is an unstable equilibrium position. However, since the system evolves under a displacement control, no failure occurs. As the system evolves along the descending branch of Fig. 11, the determinant becomes positive and increases slightly up to $20 \%$ axial strain. Then, during the force-controlled phase, $\operatorname{det} \mathbf{H}$ slowly decreases towards zero until the collapse of the grain column, as predicted in Sect. 2. It is worth noting that the determinant does not reach the zero value exactly; in fact, when approaching this value (beyond 23\% axial strain), the response of the column becomes fully dynamic. Figure 13 displays a sharp increase in kinetic energy, indicating that inertial effects can no longer be omitted, contrary to what was assumed in Sect. 2 developed in a static context. 


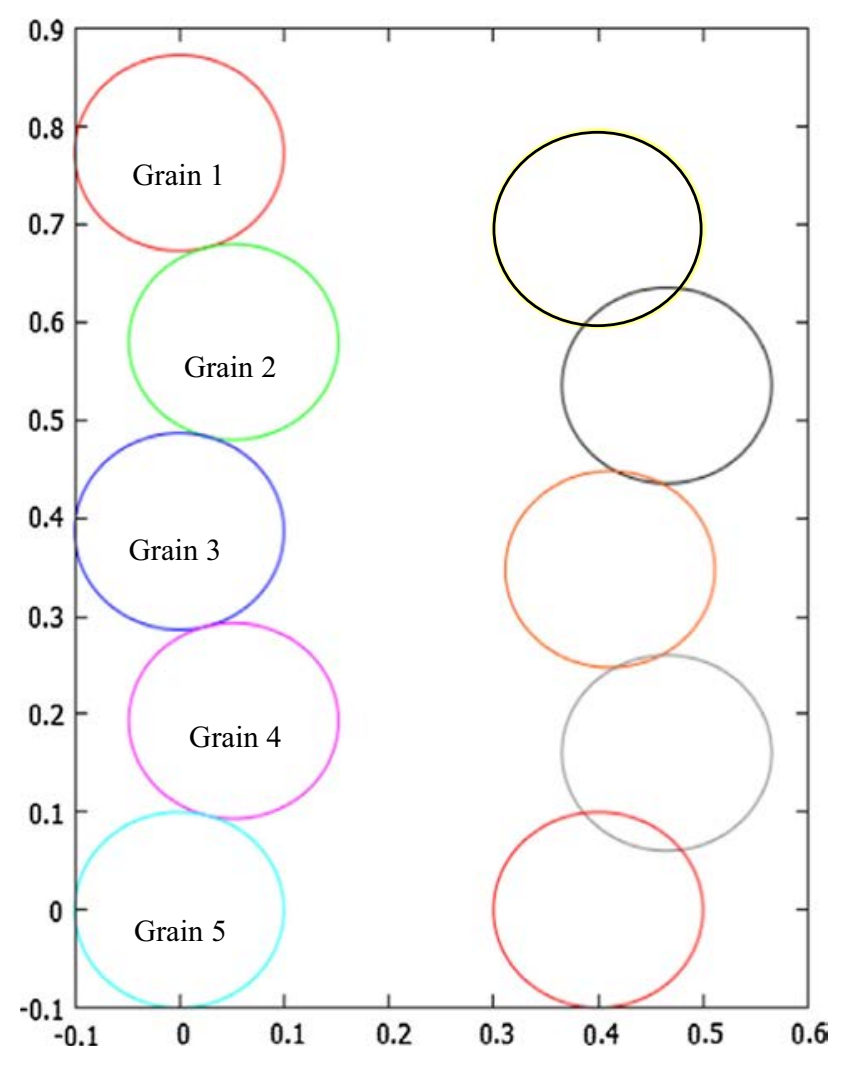

Fig. 8 Geometrical configuration of the chain before loading (left $)$ and after loading (right)

\section{Concluding remarks}

Basically, the fabric of granular materials organizes around two main components: (i) the force chains, gathering adjoining grains along a quasi-linear pattern, transmit contact forces larger than the average value; (ii) the grain loops (or clusters), surrounding the force chains, constitute the so-called weak phase and ensure the stability of these linear patterns.
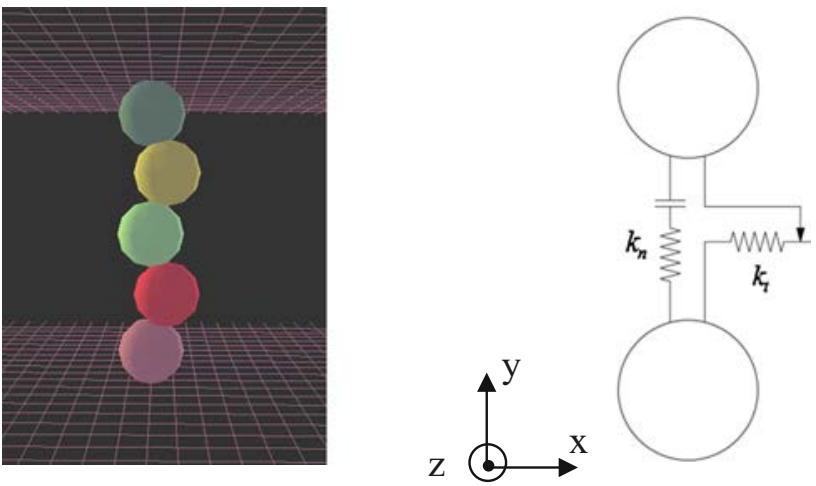

Fig. 10 YADE model and definition of the inter-particle contact law

Table 5 Numerical parameters used during the DEM simulations

\begin{tabular}{llll}
\hline Grain radius $(\mathrm{m})$ & $k_{n}(\mathrm{kN} / \mathrm{m})$ & $k_{t}(\mathrm{kN} / \mathrm{m})$ & Density $\left(\mathrm{kg} / \mathrm{m}^{3}\right)$ \\
\hline 0.1 & 1000 & 500 & 1000 \\
\hline
\end{tabular}

The linear pattern analyzed in this manuscript models a force chain, neighbored by a weak phase. The weak phase is accounted for through a set of lateral forces $G_{i}$, assumed to remain constant. In some cases (mainly due to the loading conditions, and thus, to the neighboring conditions around the chain), the axial force reaches a peak, and follows a descending branch. At the peak, the grain column is not able to sustain a higher axial force. If an additional axial load is applied to the force chain (which may occur in a granular assembly), a collapse is expected to occur, because the external axial force cannot be balanced by the internal forces developed between grains. One important feature is that this limit state does not stem from the local constitutive properties (the local behavior is purely elastic), but from the fact that the geometry of the column evolves. Of course, this instability mode is amplified if sliding occurs between neighboring grains. After developing an analytical approach in quasi-static con-

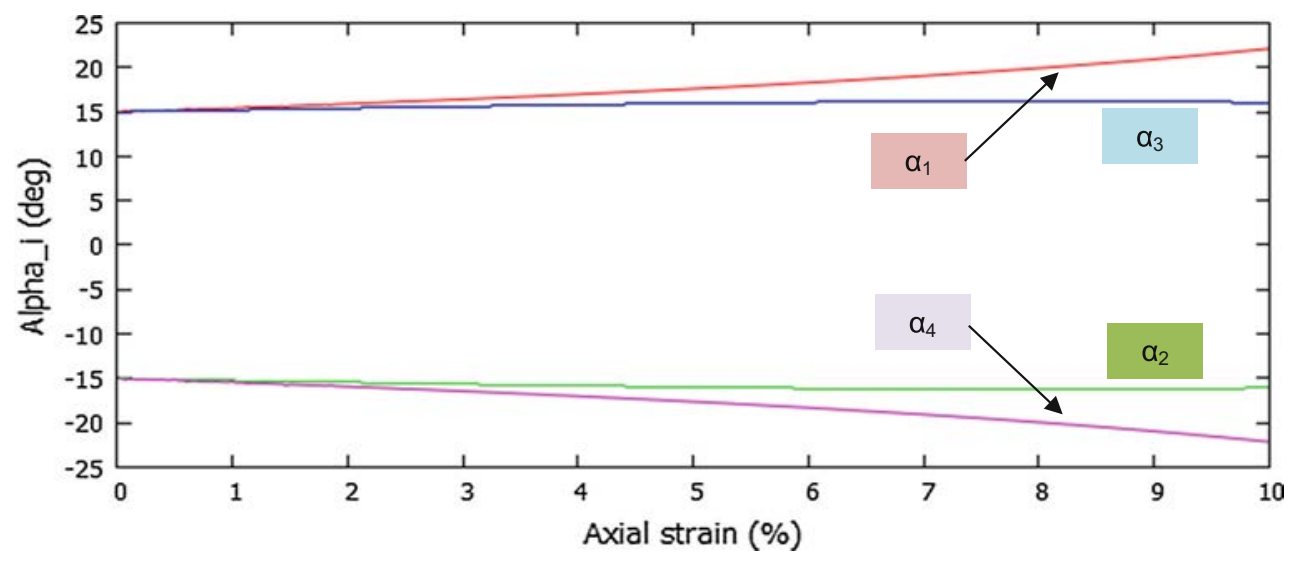

Fig. 9 Angles $\alpha_{1}, \alpha_{2}, \alpha_{3}$ and $\alpha_{4}$ versus axial strain 
Fig. 11 Axial force during the displacement-controlled phase (left of the dotted line) and during the force-controlled phase (right of the dotted line)
Fig. 12 Angles $\alpha_{1}, \alpha_{2}, \alpha_{3}$ and $\alpha_{4}$ during the displacement-controlled phase (left of the dotted line) and during the force-controlled phase (right of the dotted line)
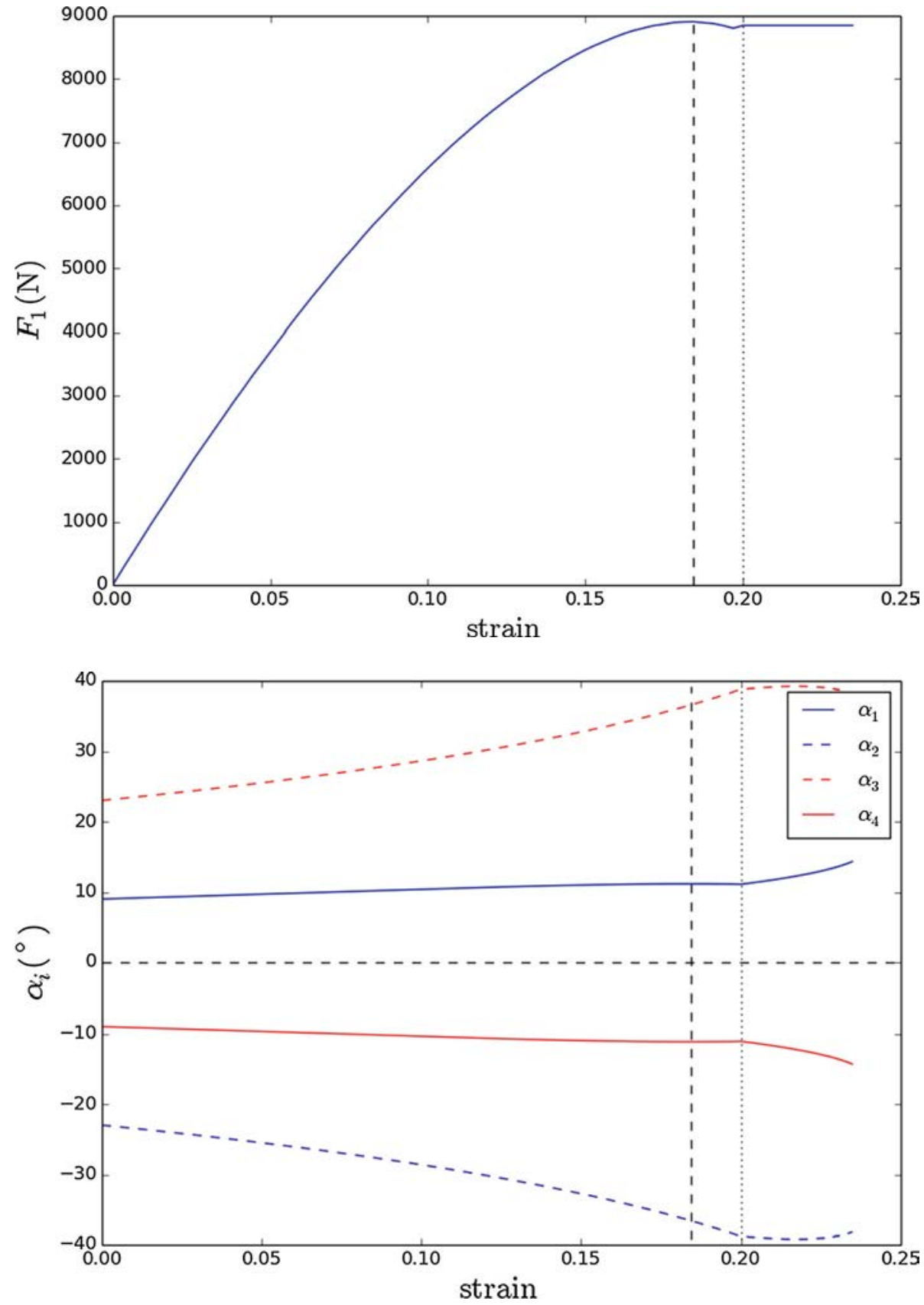

ditions, the main results were satisfactorily recovered from numerical simulations based on a discrete element model. In particular, the grain column was proved to be unstable once the axial force reached a peak. Any additional elementary axial loading makes the column collapse.

It should be emphasized that a simplified model was developed to exhibit instability modes that can occur even when the local behavior at the contact between grains is purely elastic. In this context, grain rotation and plastic sliding at contact points are both omitted. The absence of grain rotation is rather stabilizing, restricting the occurrence of instability modes. It is therefore quite challenging to observe instability in this context. Pointing out the role of translational displacements between grains with a pure elastic behavior in the occurrence of instability modes is the purpose of this manuscript.

Finally, this analysis points out the crucial interplay between force chains and adjoining clustered structures (grain clusters in 3D conditions, grain loops in 2D conditions). Adjoining clusters define the loading conditions applied to a given force chain and therefore the occurrence of possible instability modes. Interestingly, the loading conditions applied by the adjoining clusters depend on the topology of these clusters [35] and therefore on their deformability. It was clearly shown that in two-dimensional conditions, clus- 
Fig. 13 Axial strain (left) and force-controlled phase over time kinetic energy (right) during the

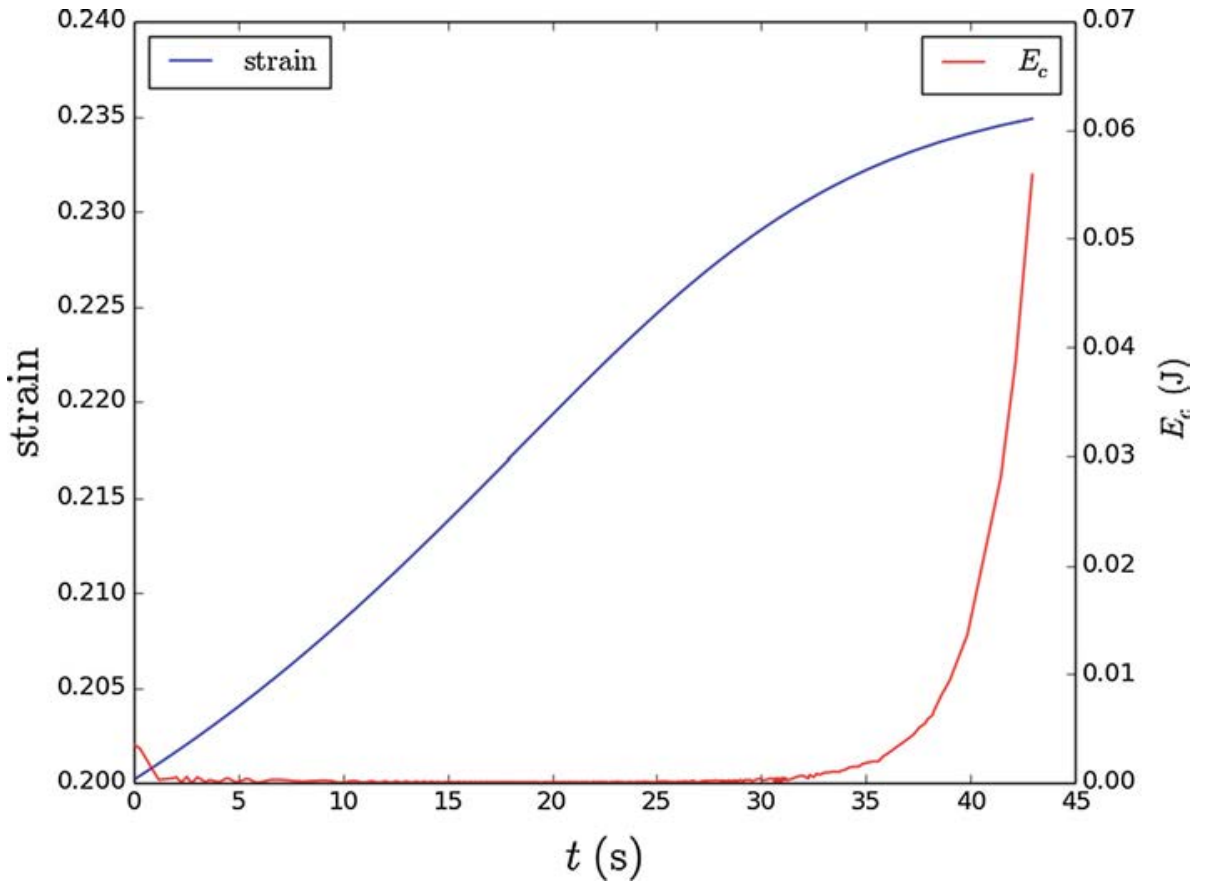

Fig. 14 Strain of the determinant of $\mathbf{H}$ during the displacement-controlled phase (left of the dotted line) and during the force-controlled phase (right of the dotted line). The inset corresponds to the force-controlled phase

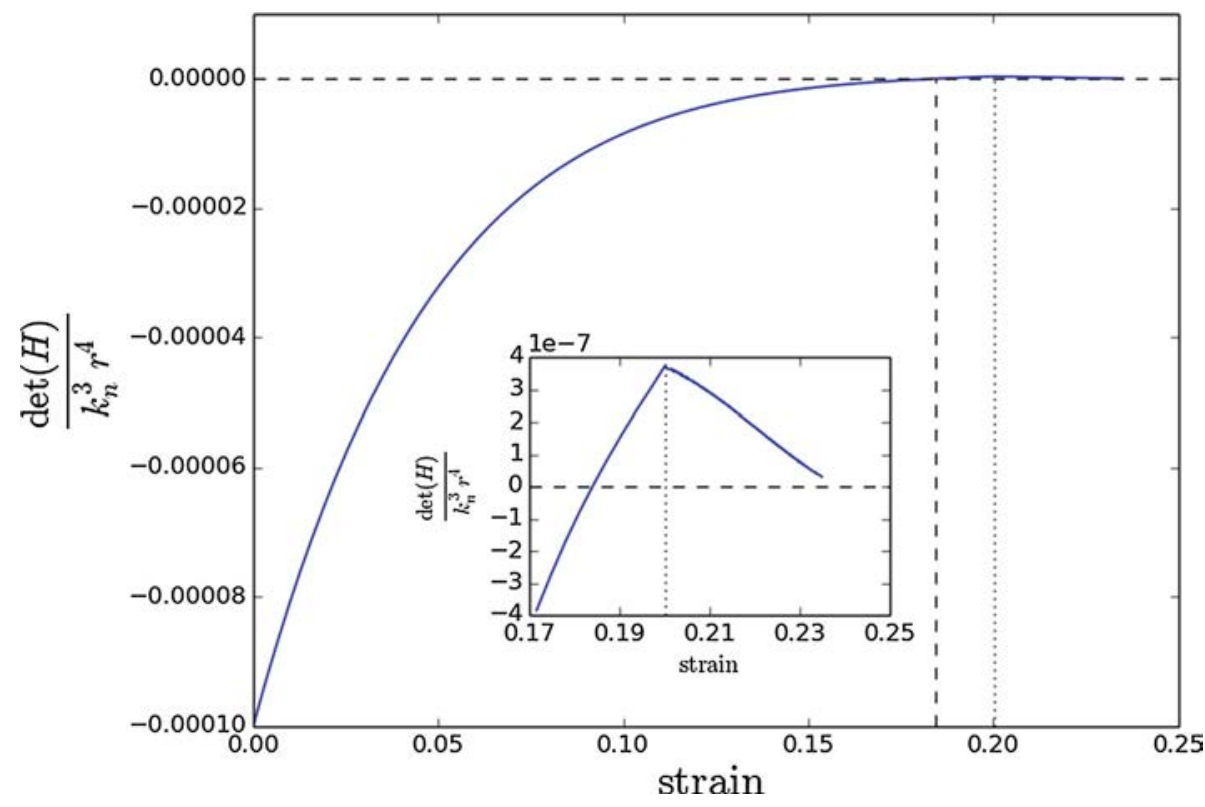

ters with at least four particles can be unstable themselves and undergo large deformations [22]. Such deformations limit the amplitude of the lateral confinement applied to the force chains, making them more prone to collapse.

It should be noted that this finding is perfectly in accordance with the recent investigation on elastic structures (Ziegler column), where it is shown that some loading conditions with specific kinematical constraints applied to an initially stable column can destabilize an elastic structure $[3,4,15,20]$.
Acknowledgements The authors would like to express their sincere thanks to the French Research Network MeGe (Multiscale and multi-physics couplings in geo-environmental mechanics GDR CNRS $3176 / 2340,2008-2015$ ) for having supported this work.

\section{Compliance with ethical standards}

Conflict of interest The authors declare that there is no conflict of interest related to this manuscript. 


\section{References}

1. Bernstein, B.: Hypoelasticity and elasticity. Arch. Rational Mech. Anal. 6, 89-104 (1960)

2. Bolotin, V.V.: Non-conservative Problems of the Theory of Elastic Stability. Pergamon Press, Oxford (1963)

3. Challamel, N., Nicot, F., Lerbet, J., Darve, F.: On the stability of non-conservative elastic systems under mixed perturbations. EJECE 13(3), 347-367 (2009)

4. Challamel, N., Nicot, F., Lerbet, J., Darve, F.: Stability of nonconservative elastic structures under additional kinematics constraints. Eng. Struct. 32(10), 3086-3092 (2010)

5. Cundall, P., Strack, O.: A discrete numerical model for granular assemblies. Geotechnique 29(1), 47-65 (1979)

6. Dantu, P.: Contribution à l'étude mécanique et géométrique des milieux pulvérulents. In: Proceedings of the 4th International Conference on Soils Mechanics and Foundation Engineering, pp. 144-148. London (1957)

7. Daouadji, A., Darve, F., Al Gali, H., Hicher, P.Y., Laouafa, F., Lignon, S., Nicot, F., Nova, R., Pinheiro, M., Prunier, F., Sibille, L., Wan, R.: Diffuse failure in geomaterials, experiments, theory and modelling. Int. J. Numer. Anal. Methods Geomech. 35(16), 1731-1773 (2011)

8. Darve, F., Servant, G., Laouafa, F., Khoa, H.D.V.: Failure in geomaterials, continuous and discrete analyses. Comp. Methods Appl. Mech. Eng. 193, 3057-3085 (2004)

9. Ericksen, J.L.: Hypoelastic potentials. Q. J. Mech. Appl. Math. 11, 67-72 (1958)

10. Hadda, H., Nicot, F., Bourrier, F., Sibille, L., Radjai, F., Darve, F.: Micromechanical analysis of second order work in granular media. Granul. Matter 15, 221-235 (2013)

11. Hamadi, K., Modaressi, A., Darve, F.: Bifurcation and instability modelling by a multimechanism elasto-plastic model. Int. J. Numer. Anal. Methods Geomech. 32(5), 461-492 (2008)

12. Hill, R.: A general theory of uniqueness and stability in elasticplastic solids. J. Mech. Phys. Solids 6, 236-249 (1958)

13. Kozicki, J., Donzé, F.: A new open-source software developed for numerical simulations using discrete modeling methods. Comput. Methods Appl. Mech. Eng. 197(49-50), 4429-4443 (2008)

14. Lerbet, J., Aldowaji, M., Challamel, N., Nicot, F., Prunier, F., Darve, F.: p-positive definite matrices and stability of nonconservative systems. Z. Angew. Math. Mech. 92(5), 409-422 (2012)

15. Lerbet, J., Kirillov, O., Aldowaji, M., Challamel, N., Nicot, F., Darve, F.: Additional constraints may soften a non-conservative structural system: buckling and vibration analysis. Int. J. Solids Struct. 50(2), 363-370 (2013)

16. Nguyen, H.N.G., Prunier, F., Djéran-Maigre, I., Nicot, F.: Kinetic energy and collapse of granular materials. Granul. Matter 18, 1-10 (2016). doi:10.1007/s10035-016-0609-1

17. Nicot, F., Darve, F.: A micro-mechanical investigation of bifurcation in granular materials. Int. J. Solids Struct. 44, 6630-6652 (2007)

18. Nicot, F., Darve, F., Khoa, H.D.V.: Bifurcation and second-order work in geomaterials. Int. J. Numer. Anal. Methods Geomech. 31, 1007-1032 (2007)
19. Nicot, F., Hadda, N., Bourrier, F., Sibille, L., Wan, R., Darve, F.: Inertia effects as a possible missing link between micro and macro second-order work in granular media. Int. J. Solids Struct. 49(10), 1252-1258 (2012a)

20. Nicot, F., Challamel, N., Lerbet, J., Prunier, F., Darve, F.: Some insights into structure instability and the second order work criterion. Int. J. Solids Struct. 49(1), 132-142 (2012b)

21. Nicot, F., Sibille, L., Darve, F.: Failure in rate independent granular materials as a bifurcation toward a dynamic regime. Int. J. Plast. 29, 136-154 (2012c)

22. Nicot, F., Veylon, G., Zhu, H., Lerbet, J., Darve, F.: Mesoscopic scale instability in particulate materials. J. Eng. Mech. 142, 04016047 (2016). doi:10.1061/(ASCE)EM.1943-7889.0001100

23. Petryk, H.: Theory of bifurcation and instability in timeindependent plasticity. In: Nguyen, Q.S. (ed.) Bifurcation and Stability of Dissipative Systems, pp. 95-152. Springer, Berlin (1993)

24. Radjai, F., Roux, S., Moreau, J.J.: Contact forces in a granular packing. Chaos 9(3), 544-550 (1999)

25. Sibille, L., Nicot, F., Donze, F.V., Darve, F.: Material instabilities in granular assemblies from fundamentally different models. Int. J. Numer. Anal. Methods Geomech. 31(3), 457-482 (2007)

26. Sibille, L., Hadda, N., Nicot, F., Tordesillas, A., Darve, F.: Granular plasticity, a contribution from discrete mechanics. J. Mech. Phys. Solids 75, 119-139 (2015)

27. Šmilauer, V., Catalano, E., Chareyre, B., Dorofeenko, S., Duriez, J., Gladky, A., Kozicki, J., Modenese, C., Scholtès, L., Sibille, L., Stránský, J., Thoeni, K.: Yade Reference Documentation. Yade Documentation (V. Šmilauer, ed.), The Yade Project, 1st ed. (2010). http://yade-dem.org/doc/

28. Tordesillas, A., Muthuswamy, M.: On the modeling of confined buckling of force chains. J. Mech. Phys. Solids 57, 706-727 (2009)

29. Tordesillas, A., Walker, D.M., Lin, Q.: Force cycles and force chains. Phys. Rev. E 81, 011302 (2010)

30. Tordesillas, A., Lin, Q., Zhang, J., Behringer, R.P., Shi, J.: Structural stability and jamming of self-organized cluster conformations in dense granular materials. J. Mech. Phys. Solids 59, 265-296 (2011)

31. Tordesillas, A., Pucilowski, S., Sibille, L., Nicot, F., Darve, F.: Multiscale characterization of diffuse granular failure. Philos. Mag. 92(36), 4547-4587 (2012)

32. Truesdell, C.: Hypoelasticity. J. Rational Mech. Anal. 4, 83-133 (1955)

33. Ziegler, H.: Principles of Structural Stability. Blaisdell Publishing Company, London (1968)

34. Zhu, H., Nicot, F., Darve, F.: Meso-structure evolution in a 2D granular material during biaxial loading. Granul. Matter 18(1), 112 (2016a). doi:10.1007/s10035-016-0608-2

35. Zhu, H., Nguyen, H.N.G., Nicot, F., Darve, F.: On a common critical state in localized and diffuse failure modes. J. Mech. Phys. Solids 95, 112-131 (2016). doi:10.1016/j.jmps.2016.05.026 